\title{
Subgrain structure and dislocations in WC-Co hard metals revealed by electron channelling contrast imaging
}

\author{
B.M. Jablon ${ }^{1,2}$, K. Mingard ${ }^{2}$, A. Winkelmann ${ }^{3}$, G. Naresh-Kumar ${ }^{1}$, B. Hourahine ${ }^{1}$, C. Trager-Cowan ${ }^{1}$ \\ 1 Department of Physics, SUPA, University of Strathclyde, Glasgow G4 0NG, United Kingdom \\ 2 National Physical Laboratory, Teddington, Middlesex, TW11 OLW, UK \\ 3 Laser Zentrum Hannover e.V., 30419 Hannover, Germany
}

\begin{abstract}
In this study, electron channelling contrast imaging (ECCI) and electron backscatter diffraction (EBSD) have been used to examine the substructure and dislocations in tungsten carbide (WC) grains in tungsten carbide-cobalt (WC-Co) hardmetals. These complimentary scanning electron microscopy (SEM) diffraction techniques provide quantifiable information of the substructure without the difficulty of transmission electron microscopy (TEM) sample preparation and examination. Subgrain structures in WC grains have rarely been reported previously because of the sample preparation difficulty, but this study has found they can occur frequently and may provide information on grain growth during sintering. $\mathrm{ECCl}$ has also shown for the first time complex dislocation networks across large grains, indicating accumulation of stress in as-sintered materials. To identify the defects revealed by ECCI more precisely, WC grains with surface normals [0001], [1 $\overline{1} 00]$ and [11̄20], were identified using inverse pole figure orientation maps generated from EBSD data. ECC images from these grains reveal defects intersecting the surface and subgrains bound by dislocations. The combination of ECCI and EBSD allows for new insights into dislocation networks in a WC-Co hardmetal sample over a large, in this case $75 \mu \mathrm{m} \times 75 \mu \mathrm{m}$, field of view.
\end{abstract}

Keywords: hardmetal, subgrain, dislocations, ECCI, EBSD, WC

\section{Introduction}

This study explores the use of the scanning electron microscopy (SEM) techniques of electron backscatter diffraction (EBSD) [1-3] and electron channelling contrast imaging (ECCI) [3-6], fast and minimally destructive techniques, for studying the microstructure and in particular the subgrain structure and defects present in tungsten carbide (WC) grains in WC-Co hardmetals (cemented carbides).

Abbreviations:

ECCl-Electron channelling contrast imaging, EBSD-Electron backscatter diffraction

TEM-Transmission electron microscopy, SEM-Scanning electron microscopy 
Hardmetals are materials with high compressive strength and wear resistance. Cobalt is used as a ductile binder phase between very hard WC grains because it is able to absorb energy and plastically deform without fracturing [7]. WC-based hardmetals are produced by a powder metallurgical route involving milling of WC and Co powders followed by liquid phase sintering. During liquid phase sintering, WC grains dissolve into the binder phase but recrystallize when the binder phase is saturated with WC and continue to crystallize and grow during initial cooling. Defects, such as dislocations, are present in the crystal lattice of WC as a result of this processing. Applications of these materials include wood processing, metal machining, electronics, and dental industries because it wears well when exposed to intermittent high impact forces, it has high compressive strength and wear resistance. Such applications are well-established, but a better understanding of the microstructure of WC, and its response to deformation, is still sought to improve performance. Part of this understanding could come from greater knowledge of the dislocation and defect structures in WC, both in the as-sintered state and as a result of plastic deformation. EBSD has been used to show levels of plastic deformation resulting from scratching [8] and hardness testing [9] but detailed imaging of dislocations and sub-grain defects has only been achieved using transmission electron microscopy (TEM) (e.g. [10-12]). High resolution transmission EM (HR-TEM) studies (e.g. [13]) have revealed fine detail of dislocation structures, but preparation and imaging of hardmetals for any TEM method is time-consuming, difficult and limited in the volume of material that it can examine. Information on the as-sintered grain structure may also provide information on the production process: studies show that the density of defects in the crystal lattice depends on the preparation of the initial WC powder [13].

In this paper we report a method that reduces the need for TEM and provides information over much larger areas by the combined use of EBSD and ECCI to study individual WC grain structures in assynthesized hardmetal grades. EBSD has been used to understand the microstructure of hardmetals in a number of studies $[8,9,14]$ which explain the use of this SEM based diffraction technique to determine crystal orientation. In brief, in EBSD the sample is tilted at around $70^{\circ}$ to the normal of the incident electron beam. The impinging electrons are scattered inelastically through high angles forming a diverging source of electrons which can be diffracted. The resultant electron backscatter diffraction pattern (EBSP) consists of a large number of overlapping bands, known as Kikuchi bands, which are closely related to a 2-D projection of the crystal structure. Acquiring EBSPs over a grid of points on a sample allows mapping of the sample's microstructure. 
$\mathrm{ECCl}$ is also an SEM based diffraction technique which can be used to detect small orientation changes or changes in lattice constant in a material. ECCI micrographs may be produced when a sample is placed so that a plane or planes are at, or close to, the Bragg angle with respect to the incident electron beam. Any deviation in crystallographic orientation or in lattice constant due to local strain will produce a variation in contrast in the resultant $\mathrm{ECCl}$ micrograph. Extremely small changes in orientation and strain are detectable, revealing subgrains and extended defects such as dislocations and stacking faults [3-6]. We can also investigate the contrast exhibited by a defect for different diffraction conditions [15,16]; ultimately identifying the nature of the defect. To date, there does not however appear to have been any published work on the use of ECCl to identify dislocation types and substructures in hardmetals.

For ECCI and EBSD a flat sample surface free of preparation damage is required. Consequently, we discuss issues of specimen preparation required to reveal the presence of subgrains, intra-granular dislocations and stacking faults in the WC grains. Data was collected from WC grains with surface normals [0001], [1 $1 \overline{1} 00]$ and [11 20$]$. We investigated the subgrain structure, distributions of dislocations and their relationship with subgrains and estimated the density of vertical threading dislocations for each grain. We also discuss how ECCI may be used to explore the nature of dislocations present in the WC grains.

\section{Materials and Methods}

In the present work, results were obtained from two grades of hardmetal, a coarse grained 11wt.\% Co (mean grain size (equivalent circle diameter) $4.5 \mu \mathrm{m}$ ), 11C, and a medium grained 10wt\% Co (mean grain size $2.1 \mu \mathrm{m}$ ), 10M. The samples were first mechanically polished to an optical finish using standard metallographic techniques, finishing with a colloidal silica polish which leaves a mirror finish and no visible scratches on the surface. Samples were also further polished in a Hitachi IM4000 argon ion beam polishing system to try to remove any fine scratches due to the mechanical polishing. In general significant material removal by ion beam polishing required an $\mathrm{Ar}+$ ion beam of $6 \mathrm{kV}$ for a minimum of 30 minutes, followed by lower beam energies of 3 or $1.5 \mathrm{kV}$ for similar times. This created a polished surface ready for examination in the SEM. While producing a scratch free surface, ion beam polishing induces shallow (of the order of $10 \mathrm{~nm}$ ) dimples in the WC grains despite rotation under the ion beam to even out variation in milling rate with grain orientation: these dimples need to be taken into account when analyzing the acquired data. 
In this study, ECCl images were acquired at $20 \mathrm{keV}$ and a $10 \mathrm{~mm}$ working distance in the backscatter geometry in a Zeiss Auriga FIB SEM using the Zeiss supplied quadrant diode backscattered electron detector which is inserted beneath the pole piece. The sample was tilted by around $20^{\circ}$ away from the horizontal to increase the intensity of the backscattered signal, as the number of backscattered electrons increases as you increase the tilt of the sample. A series of images of single WC grains with the sample at different tilts or for different rotations were then acquired. In the case of the tilt series, $\mathrm{ECCl}$ images were acquired for tilts between $17^{\circ}$ and $22^{\circ}$ with the sample tilt changed by $0.2^{\circ}$ between image acquisitions. For the rotation series, the sample was tilted at $20^{\circ}$ and rotated $10^{\circ}$ between image acquisitions, with the sample rotated by $180^{\circ}$ for each grain. At the time of measurement it was not possible to precisely determine the diffraction conditions for each image acquisition. Diffraction conditions can be determined through the acquisition of local area electron channelling patterns, but this requires local "rocking" of the beam [3] which was not possible in the SEM used for acquisition of this data set. For this work the diffraction conditions for a few of the ECCI micrographs were estimated from the grain orientation with respect to the electron beam. An alternative technique, rotational-electron channelling contrast imaging [17], has been used to determine orientation conditions when imaging dislocations in an SEM. A more precise determination of diffraction conditions for a range of ECCI micrographs for identification of individual defects will be the subject of future work.

EBSD data in this study was collected in a Zeiss Auriga FIB SEM outfitted with an Oxford NordlysNano system. EBSD maps were acquired at 20 or $30 \mathrm{kV}$ accelerating voltage with a $10 \mathrm{nA}$ beam and the sample tilted at $70^{\circ}$ from the horizontal.

\section{Results}

\subsection{ECCl and EBSD}

Initial studies were carried out on a randomly oriented grain in the $10 \mathrm{M}$ grade which had only a very short (10 minute) ion beam polish. Figure 1 shows a conventional secondary electron image of the sample acquired at $20 \mathrm{kV}$, tilt corrected for the $70^{\circ}$ tilt applied to enable mapping by EBSD. Individual WC grains can be identified but the only contrast variation is between the WC and the binder, and not between different grains. 


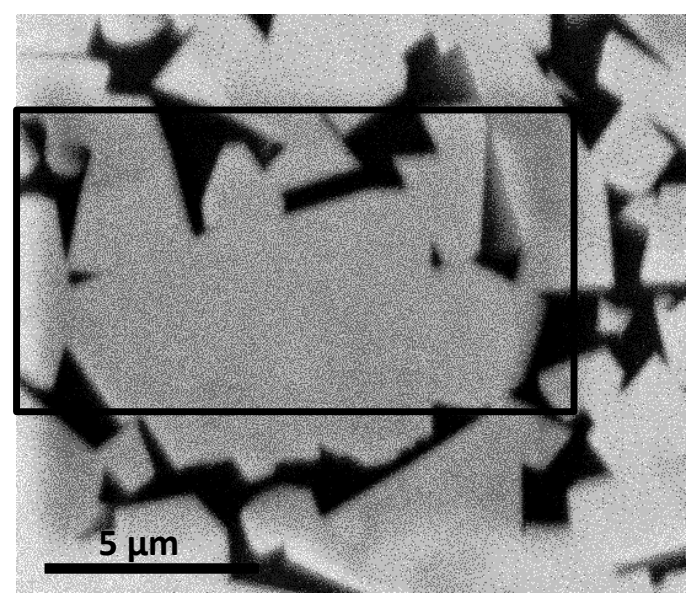

Figure 1. Secondary electron image of $10 \mathrm{wt} . \%$ Co sample. Very little detail of structure within the bright WC grains is visible; the area mapped by EBSD in Figure 2 is indicated by the black rectangle.

The area around the large grain was mapped by EBSD at $20 \mathrm{kV}$ using a relatively long $50 \mathrm{~ms}$ acquisition time and a resolution of $640 \times 480$ pixels for the EBSD patterns to improve angular accuracy. This slow acquisition allowed good patterns to be acquired, but allowed time for drift of the sample upwards (relative to the map orientation, and opposite to the direction of data acquisition). This caused compression of the target area of $240 \times 170$ pixels at a $60 \mathrm{~nm}$ step size to give an average pixel size in the vertical direction of approximately $34 \mathrm{~nm}$ and only partial mapping of the grain. The EBSD maps shown in Figure 2 are corrected for the drift and were scaled to the electron images which have negligible drift. All the EBSD maps of Figure 2 were produced from the EBSD data using MTEX [18-20], where the orientation of each EBSP was determined by pattern matching to dynamical simulations [21]. 

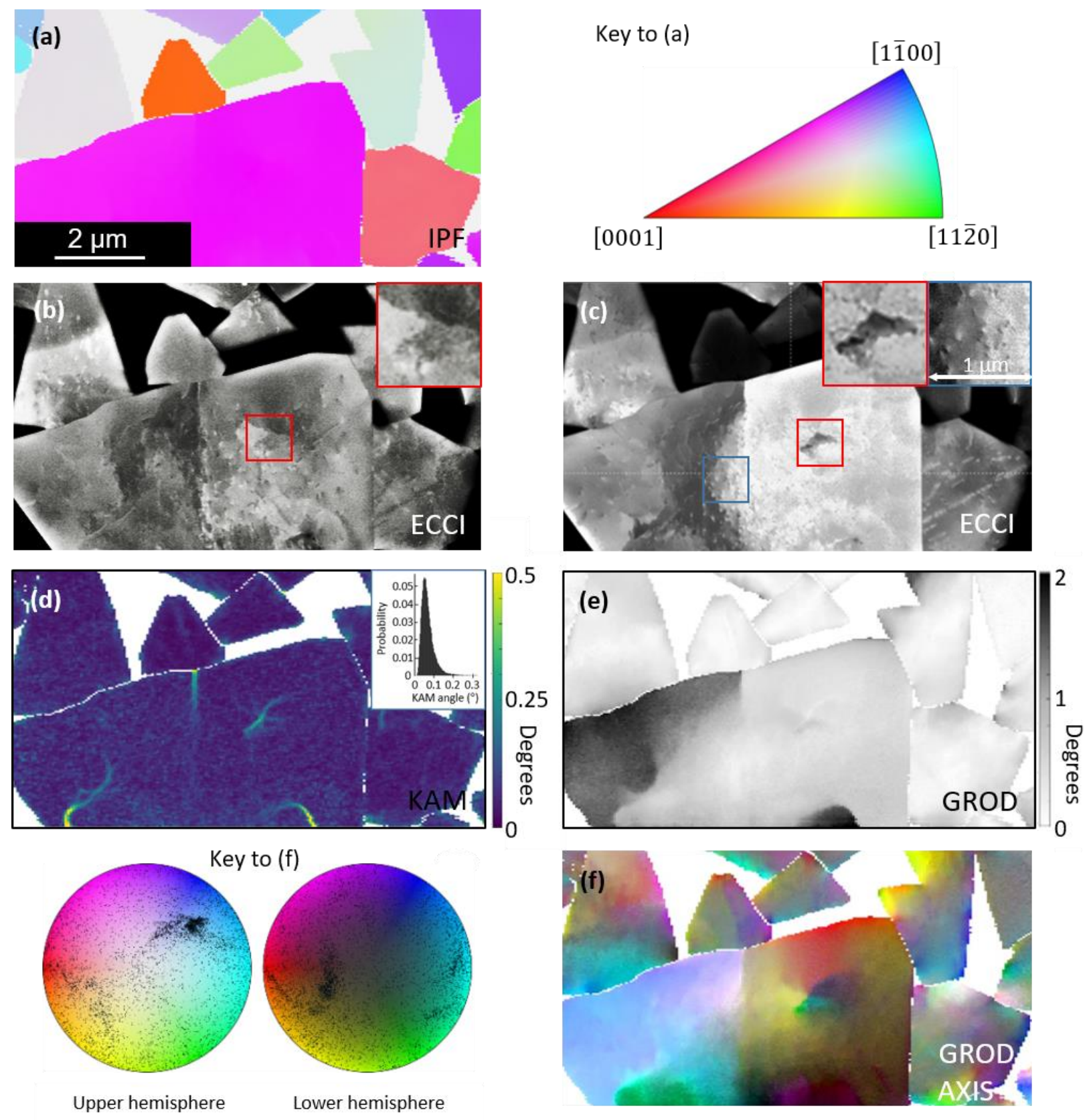

Figure 2.

(a) Orientation map coloured with a z-axis inverse pole figure colour scale.

(b) $\mathrm{ECCl}$ image of the same area as (a), sample was by tilted $20.4^{\circ}$ from the horizontal.

(c) ECCl image of the same area as (a), sample was by tilted $17^{\circ}$ from the horizontal. The inset shows an expanded view of the area delineated by the blue box in the main image, and highlights the dislocations revealed by ECCI. The red insets shows an expanded view of the area delineated by the red boxes in the main images of (b) and (c), and highlight subgrains revealed by ECCI.

(d) Kernel average misorientation (KAM) map. The inset shows the distribution of KAM angles.

(e) Grain reference orientation deviation (GROD) map. Each pixel has a value (in degrees) equal to the disorientation that pixel has with the mean orientation of each grain.

(f) Grain reference orientation deviation axis (GROD axis) map. Each pixel has a value denoting the rotation axis with respect to the mean, as defined by the key to the left of the image. The black dots in the key denote the directions for each point on the map.

Maps (a), (d), (e) and (f) were produced from the EBSD data using MTEX, where the orientation of each EBSP was determined by pattern matching to dynamical simulations. 
Before comparing the EBSD maps with the $\mathrm{ECCl}$ images in Figure 2, a significant point to note is that the EBSD maps took approximately 40x longer to acquire. The most important effect of this longer capture time is the drift discussed earlier and the resultant image distortion. After adjustment of the EBSD maps to compensate for the drift, useful information can be obtained from comparison of the EBSD maps and $\mathrm{ECCl}$ images.

Figure 2a shows a 6/mmm inverse pole figure (IPF) map. IPF maps represent crystallographic orientation in terms of the sample coordinate system. This map does not reveal any variation within each WC grain and simply illustrates that this is a polycrystalline sample with grains oriented in different directions.

For comparison, Figures $2 \mathrm{~b}$ and $2 \mathrm{c}$ show two ECCl images of the same region as Figure 1 and $2 \mathrm{a}$, acquired with relative tilts of $20.4^{\circ}$ and $17^{\circ}$ respectively from the horizontal. A significant level of additional information on the substructure in the grains is revealed by ECCI. Distinct subgrains can be seen in the large grain, for example the red insets in Figure $2 \mathrm{~b}$ and $2 \mathrm{c}$, as well as individual dislocations, blue inset in Figure 2c. Networks of dislocations are also revealed, which suggest a gradual rotation of the lattice across distances of the order of $1 \mu \mathrm{m}$. Subgrains and dislocations change contrast and appear and disappear in the ECCl images as the sample is tilted and the diffraction conditions are changed. In order to reveal all of the subgrains and crystal lattice defects in a grain, a series of ECC images must be acquired to capture all the lattice distortions within the grain.

The IPF colour scale of Figure $2 \mathrm{a}$ is insensitive to the orientation changes within grains revealed by $\mathrm{ECCl}$, but some quantification is possible by further analysis of the EBSD data. Figure $2 \mathrm{~d}$ shows a Kernel Average Misorientation (KAM) map where the KAM angle is the average misorientation angle from the map point to each neighbor [22]. The KAM map identifies some of the subgrain boundaries of the subgrains revealed by $\mathrm{ECCl}$ where the change in orientation across them is greater than $\approx 0.2^{\circ}$.

Figures 2e shows a grain reference orientation deviation (GROD) map. In this case each pixel has a value (in degrees) equal to the disorientation that pixel has with the mean orientation of the grain in which the pixel lies. Figure $2 f$ shows a grain reference orientation deviation axis (GROD axis) map. In this case each pixel has a value denoting the rotation axis with respect to the mean, as defined by the colour key to the left of the image. The colour key indicates the direction of the misorientation axes in real space above (upper hemisphere) or below (lower hemisphere) the sample. The black dots indicate these directions for each point in the map. The GROD axis map provides significantly more information on the 
misorientations in the grain when compared to the IPF map. While not providing the high level of detail observed in the ECCl images, these EBSD maps provide a quantitative measure of the misorientations. Colour changes in the GROD axis map match with the subgrain boundaries in the KAM and those observed in the ECCl images. The colour scale also reveals gradual changes in orientation within the subgrains.

\subsection{Preliminary Dislocation Analysis}

In the example shown in Figure 2, the grain observed was chosen randomly and the ECCl imaging sequence was obtained by tilting in $0.2^{\circ}$ increments. Within the limitations of the angular accuracy of the EBSD system, it was not possible to determine the diffraction conditions precisely enough to analyse the dislocation structures in detail. Further detailed imaging was therefore carried out on grains of known orientation. This was achieved by first acquiring EBSD maps of $400 \times 300 \mu \mathrm{m}$ areas, such as the one shown in Figure 3a. Figure 3a is an EBSD pattern quality map, a map that reflects the "quality" or "sharpness" of the diffraction bands in the EBSP acquired at each pixel. Grains with simple orientations, that is grains whose normals lie close (within $3-5^{\circ}$ ) of one of the principal crystallographic axes [0001], [11̄00], [11 $\left.\overline{2} 0\right]$, were identified from inverse pole figures such as the one shown in Figure $3 b$. Figure $3 b$ shows those grains whose normals are close to [1 $1 \overline{1} 00]$. These grains are coloured blue in the EBSD quality map of Figure $3 a$. The crystal orientation of a [11 00$]$ grain (circled in orange in Figure 3a) is depicted in Figure 3c. A higher resolution image of this [1 $1 \overline{100}]$ oriented grain is shown in Figure 4.
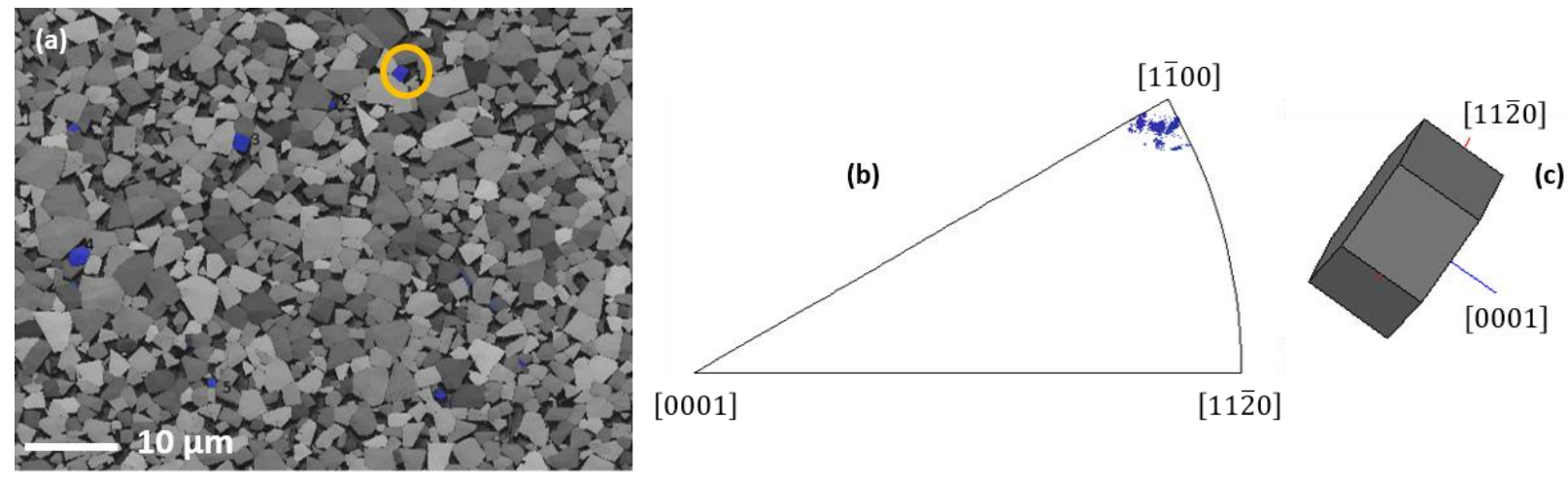

Figure 3. Identifying grains close to the $[1 \overline{100}]$ sample normal for hexagonal tungsten carbide grains. (a) A large area EBSD pattern quality map where the grains coloured blue are those identified with the IPF pole figure as having a sample normal close to [1100], (b) IPF pole figure highlighting grains whose principal crystallographic are close to the sample normal.

(c) The orientation of the grain circled in orange in Fig. $3 a$. 
On identifying grains with the [0001], [11 00$]$ and [1120] axes normal to the sample, series of $\mathrm{ECCl}$ rotation images were then acquired for each of these grain types which were free of polishing damage. Figure 4 shows examples of 3 grains from the $11 \mathrm{E}$ grade, respectively oriented with the [0001], [1100], [1120], axes normal to the sample. Dislocations are revealed in the ECCI micrographs intersecting the surface: examples are indicated by arrows in Figure 4. The number of dislocations intersecting the surface in each rotation series was counted per rotation image to calculate the vertical threading dislocation density per grain.. Densities were similar in all three grains: $\approx 4 \times 10^{8} \mathrm{~cm}^{-2}$ threading dislocations in the [0001] oriented grain, and $\approx 2 \times 10^{8} \mathrm{~cm}^{-2}$ in $[1 \overline{1} 00]$ and $[11 \overline{2} 0]$.
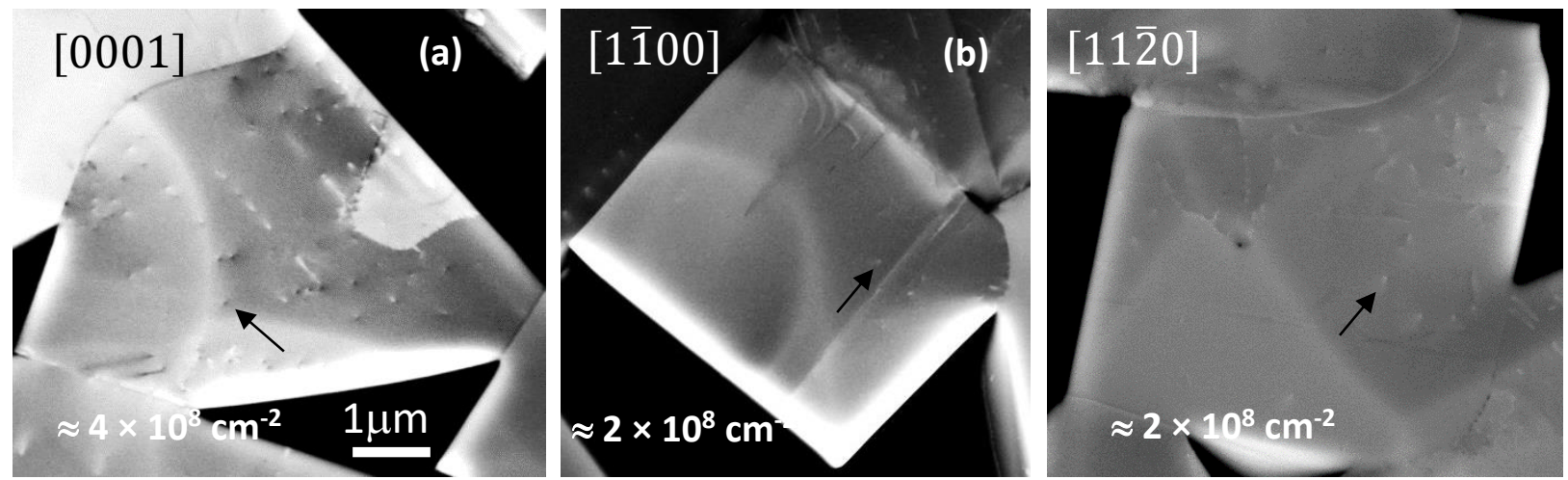

Figure 4. Dislocations as revealed by ECCI in (a) [0001] oriented grain, (b) [1 $\overline{100}]$ oriented grain, (c) $\lceil\mathbf{1 1} \overline{2} 0\rceil$ oriented grain. (The scale bar is the same for all three grains.)

Figure 5 shows three images from a rotation series acquired from the [1120] oriented crystal shown in Figure 4c. The facets visible on the grain were within $0.2^{\circ}$ of being perpendicular to each other, which would be expected for such an orientation and gives some degree of confidence in assigning the [1100] and [0001] directions determined from EBSD mapping to be parallel to the edges of the grain. The images in Figure 5 have been aligned so that they are parallel with the middle image (Figure 5b). This image is labelled as $0^{\circ}$ since it was acquired with the grain edges parallel and perpendicular to the image edge and thus to the scanning direction. Thus to a first approximation, the diffraction g-vector selected was close to [1100]. The image on the right Figure $5 \mathrm{c}$ was acquired with the sample rotated at $90^{\circ}$ with respect to the middle image, and thus to a first approximation, the selected g-vector was close to (0002). The left hand image Figure 5 a was acquired for the crystal at $-20^{\circ}$ from the middle image and represents a random unknown diffraction condition. 


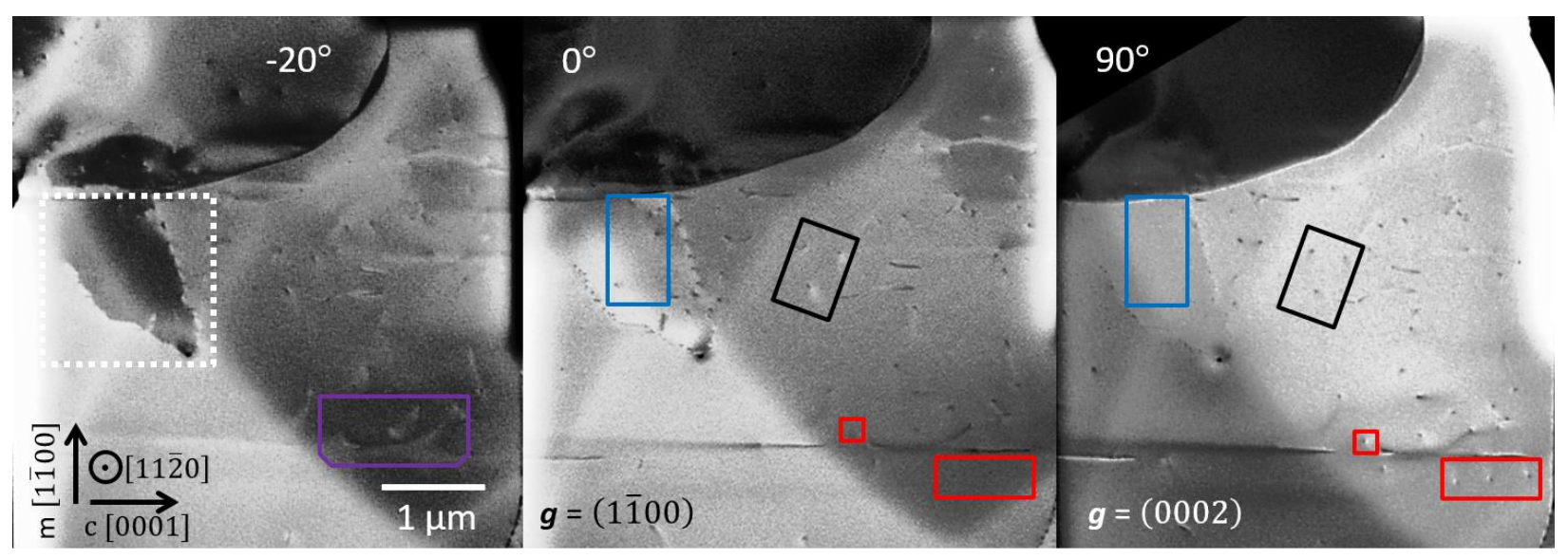

Figure 5. Dislocations as revealed by ECCI in a [11 $\overline{2} 0]$ oriented grain.

\section{(a) $-20^{\circ}$ rotation (b) $0^{\circ}$ rotation (c) $90^{\circ}$ rotation. The significance of the coloured boxes is} discussed in the text.

A subgrain is visible in the top left of image Figure 5a (outlined by the dashed white box) but shows no contrast relative to the parent grain in the middle Figure $5 \mathrm{~b}$ and right Figure $5 \mathrm{c}$ images. Euler angle calculations from EBSD (Table 1 ) indicate a very small change of its orientation relative to the parent grain $\left(0.1-0.2^{\circ}\right.$ around all three axes).

\begin{tabular}{|c|c|c|}
\hline Euler angle $\left({ }^{\circ}\right)$ & Subgrain & $\begin{array}{l}\text { Parent } \\
\text { grain }\end{array}$ \\
\hline$\phi_{1}$ & 0.8 & 0.7 \\
\hline$\Phi$ & 92.8 & 93 \\
\hline$\phi_{2}$ & 59.1 & 59 \\
\hline
\end{tabular}

Table 1: Euler angles for large (parent) grain and subgrain (outlined by dashed white box in Figure 5)

Close inspection of the dislocations contained within the subgrain shows that there are a number of dislocations visible in the middle image (Figure $5 \mathrm{~b}$ ) which are not visible in the right-hand image (Figure $5 c)$, these are highlighted by the blue box in Figure $5 \mathrm{~b}$ and $5 \mathrm{c}$. There are also dislocations which appear in the right-hand image (Figure 5c) but are very faint in the middle image (Figure 5b), these are highlighted by the red boxes in Figure $5 \mathrm{~b}$ and $5 \mathrm{c}$. There are also dislocations which appear in both the middle and right-hand images and these are highlighted by the black box in Figure $5 \mathrm{~b}$ and $5 \mathrm{c}$. We have used these observations to make a first, very tentative identification of these dislocations by applying the $\boldsymbol{g} \cdot \boldsymbol{b}$ invisibility criterion used in transmission electron microscopy, where $\boldsymbol{g}$ is the diffraction vector and $\boldsymbol{b}$ is 
the Burgers vector of the dislocation [23]. In simple terms, dislocations are invisible in an ECCl image if they do not distort the plane(s) which diffract the incident electron beam. In such a diffraction condition, the scalar product $\boldsymbol{g} \cdot \boldsymbol{b}=0$. This is only strictly true for screw dislocations where the Burgers vector of the dislocation and the dislocation line direction are parallel. For edge and mixed dislocations the condition $\boldsymbol{g} \cdot \boldsymbol{b} \times \boldsymbol{u}$, where $\boldsymbol{u}$ is the dislocation line direction, also needs to be satisfied. However the visibility of both edge and mixed dislocations are considerably reduced if $\boldsymbol{g} \cdot \boldsymbol{b}=0$. A further factor which needs to be taken into account when attempting to apply the invisibility criterion to ECCI images (and plan view TEM images), is the effect of surface relaxation on the observed defect contrast. The variation in strain due to surface relaxation around a defect can dominate the observed defect contrast [24]. However this may not be an issue in WC due to the hardness of this material.

The dislocations in the blue box, visible in the middle image (Figure 5b), but not visible in the righthand image (Figure 5c) (blue box) satisfy the condition $\boldsymbol{g} \cdot \boldsymbol{b} \neq 0$ when $\boldsymbol{g}$ is (1100) and $\boldsymbol{g} \cdot \boldsymbol{b}=0$ when $\boldsymbol{g}$ is (0002). This observation is consistent with these dislocations being mixed dslocations with $\boldsymbol{b}=1 / 3[\overline{1} 2 \overline{1} 0]$ or $\boldsymbol{b}=1 / 3[\overline{2} 110]$ (See Table 2) [25]. Similar analysis can be applied to the dislocations highlighted by the red boxes: $\boldsymbol{b}=[0001]$ (edge) or $\boldsymbol{b}=1 / 3[11 \overline{2} 3]$ ) (mixed) and to the dislocations highlighted by the black box $b=1 / 3[\overline{1} 2 \overline{1} 3]$ ) (mixed) or $b=1 / 3[\overline{2} 113])$ (mixed). Both (hkil) (4 indices) and (hkl) ( 3 indices) and [UVTW] (4 indices) and [uvw] ( 3 indices) are listed to describe $\boldsymbol{g}$ and $\boldsymbol{b}$ respectively in Table 2, where the dot product $\boldsymbol{g} \cdot \boldsymbol{b}$ is calculated using (hkl) and [uvw] respectively. Note that there are no stacking faults observed in the grain shown in Figure 5 as these would satisfy the requirement for invisibility when $\boldsymbol{g}=(0002)$.

\begin{tabular}{|l|l|l|l|}
\hline & $\begin{array}{l}\text { Blue box } \\
\boldsymbol{b}=1 / 3[\overline{1} 2 \overline{1} 0]([030]) \\
\text { mixed dislocations or } \\
\boldsymbol{b}=1 / 3[\overline{2} 110]([\overline{3} 00]) \\
\text { mixed dislocations }\end{array}$ & $\begin{array}{l}\text { Red boxes } \\
\boldsymbol{b}=[0001]([001]) \\
\text { edge dislocations or } \\
\boldsymbol{b}=1 / 3[11 \overline{2} 3]([333]) \\
\text { mixed dislocations }\end{array}$ & $\begin{array}{l}\text { Black } \boldsymbol{b o x} \\
\boldsymbol{b}=1 / 3[\overline{1} 2 \overline{1} 3]([033]) \\
\text { mixed dislocations or } \\
\boldsymbol{b}=1 / 3[\overline{2} 113]([\overline{3} 03]) \\
\text { mixed dislocations }\end{array}$ \\
\hline$(1 \overline{1} 00)(1 \overline{1} 0)$ & $\boldsymbol{g} \cdot \boldsymbol{b} \neq 0$ (visible) & $\boldsymbol{g} \cdot \boldsymbol{b}=0$ (invisible) & $\boldsymbol{g} \cdot \boldsymbol{b} \neq 0$ (visible) \\
\hline$(0002)(002)$ & $\boldsymbol{g} \cdot \boldsymbol{b}=0$ (invisible) & $\boldsymbol{g} \cdot \boldsymbol{b} \neq 0$ (visible) & $\boldsymbol{g} \cdot \boldsymbol{b} \neq 0$ (visible) \\
\hline
\end{tabular}

Table 2: Summarising $\boldsymbol{g} \cdot \boldsymbol{b}$ analysis for grain shown in Figure 5. $g$ and $\boldsymbol{b}$ are given in both 4 index and 3 index notation. 
More work needs to be done to understand all the defects revealed by these images, for example curved dislocations like the one highlighted by the box in Figure 5a, but these simple observations have allowed us to do some initial, if crude, dislocation and stacking fault analysis of the observed defects.

\section{Discussion}

\subsection{Dislocations}

Burgers vectors identified in Figure 5 are consistent with previous observations of WC [10,26,27], when it is considered that the samples are in the as-received state and not, as more commonly considered after deformation for which stacking faults and their associated partial dislocations are more commonly reported $[10,13,26]$. Early work assessed slip around hardness indents to determine possible slip systems and noted that it was uncertain what dislocations were formed by the deformation and what were present in the as received/polished state [27]. Clearly the current technique now provides the potential for dislocations to be identified before and after indentation, if the current work is combined with more precise angular measurement than is possible by EBSD by combining with SACP to enable robust determination of the diffraction vector for any given orientation [6].

\subsection{Sample Preparation}

As with other techniques such as TEM foil analysis, care has to be taken that dislocations observed are inherent in the material and not an artefact produced by the sample preparation method. Dislocations produced by scratches from the coarser stages of mechanical polishing can be very difficult to identify, particularly as the scratches may themselves have been removed to leave a flat surface. An example of this is seen in Figure $2 c$ in which a strong line can be seen running across the width of the grain to the right of the large central grain, with a few shorter lines parallel to it; along with a further line at about $60^{\circ}$ to it. The perfectly straight nature of these lines suggest they are probably the residual effects of scratching damage during an earlier polishing stage which was not removed by the final stage; because hardness varies strongly with orientation [9] scratches often remain in grains presenting a harder orientation to the surface than in the softer orientations.

A prolonged final polishing stage with colloidal silica can eliminate most scratches but this generally leads to significant differential polishing of the Co and edge rounding on the WC grains. This in turn results in high contrast variation in $\mathrm{ECCl}$ and obscures information around the edges of the grain. The ion beam polishing used on the samples in Figures 4 and 5 does appear to eliminate residual scratches with a high 
degree of confidence but suffers from producing an irregular wavy surface. This wavy surface produces confusing contrast effects such as the curved arcs and ridges seen in Figures 5a-c, although no evidence was observed to suggest it might itself nucleate dislocations at the ridges formed or elsewhere on the surface.

\subsection{Sub Grain structure}

The grain observed in the 10wt.\% Co grade in Figure 2 has a complex sub structure with multiple subgrains. The $11 \mathrm{wt} . \%$ Co grade in general appears to have a less complex structure but many grains still show distinct sub grains (Figs $4 \mathrm{a}$ and 5a). A lower magnification ECCl image shown in Figure 6 reveals at least 4 subgrains with clear boundaries, circled in yellow (including the one shown in Figure 4a, circled in a broken yellow line), and a further 3 more diffuse regions of misorientation in red boxes. The 2D nature of the images cannot show the full detail of the location of the subgrains relative to all the external boundaries of the enclosing grain in 3D, but 4 of the 5 well defined sub grains have a WC-Co boundary in common with the enclosing grain. Only one (the bottom of the 4 in Figure 6 ) is fully enclosed within the centre of a WC grain, and it is not possible to tell if it has a common WC-Co boundary above or below the plane of the image.

Two questions are posed by these observations. Firstly, are the sharply defined subgrains the result of grain growth from the subgrain during liquid phase sintering, or the result of absorption of the subgrain by a neighbouring grain of almost identical orientation? Secondly, are the more diffuse subgrains the result of grain growth, or the result of accommodation of stresses during cooling from the sintering temperature? 


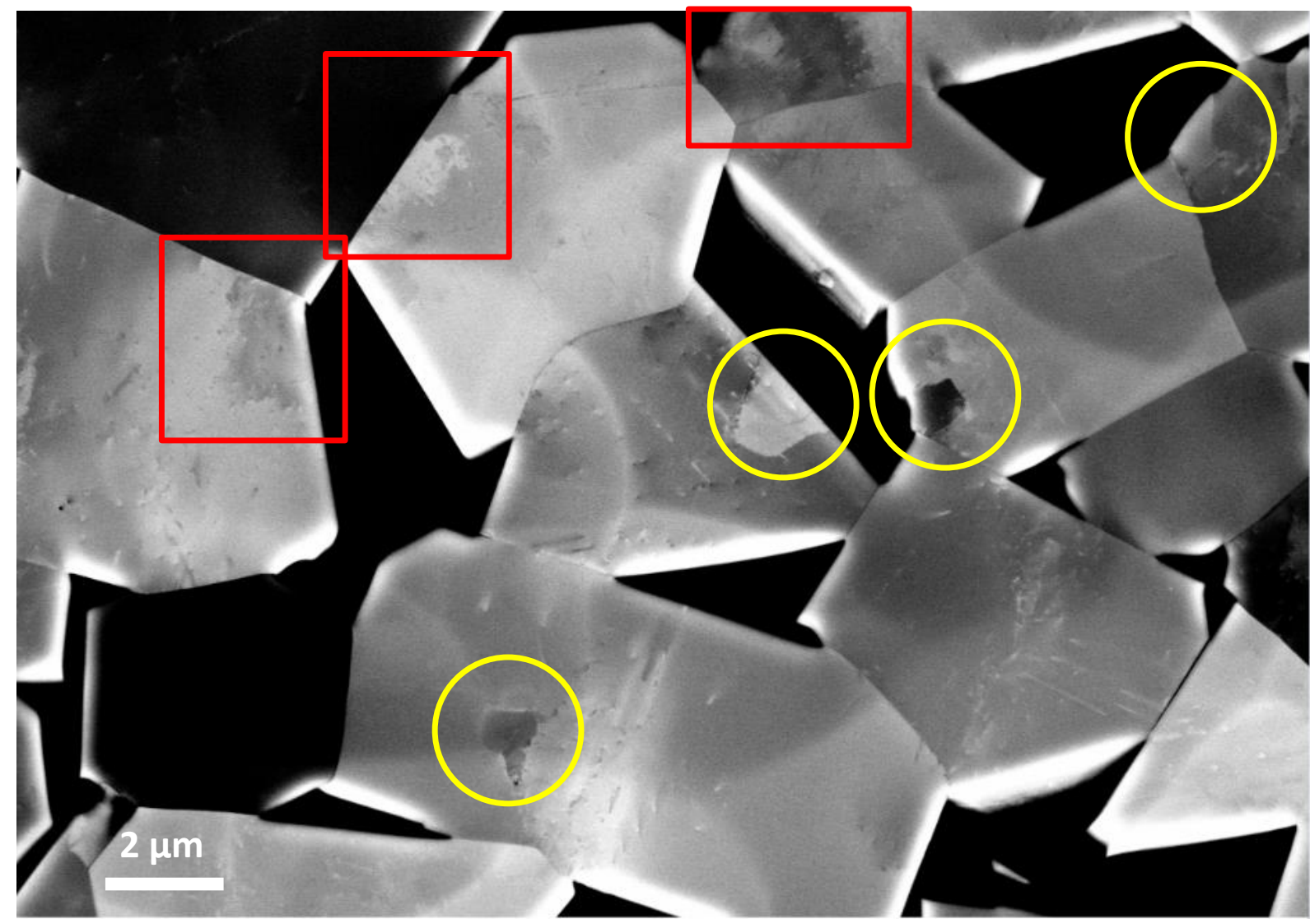

Figure 6. Low magnification $\mathrm{ECCl}$ image taken at one $\mathrm{ECCl}$ diffraction condition reveals 4 subgrains with clear boundaries, circled in yellow, and a further $\mathbf{3}$ more diffuse regions of misorientation in red boxes. The dotted yellow circle indicates the subgrain revealed in figure $4 a$.

To the best of the authors' knowledge, only one previous observation has been reported of subgrains present within WC grains in a WC based hardmetal. Delanoe et al (2007) [28] observed by TEM one grain enclosing complete 3 smaller grains and a second grain "partly engulfing"a smaller grain at a WC-Co boundary. However misorientation in these cases were $\approx 2^{\circ}$, or 3 times larger than the maximum observed here or were actually $\Sigma 2$ coincidence site lattice misorientations. It was suggested the grains were absorbed by abnormal grain growth, whereas the observations here are of grains well within the normal grain size distribution.

A number of studies have investigated the evolution of WC grain shape and size in relationship to the starting powders (e.g. [11,29-32]). In several cases evidence was found that WC grains develop facets, even in the solid state where WC-Co boundaries develop [33], and that the facets affect the growth rates of the grains. However none report on the sub structure or show any evidence for growth from a 
nucleating grain, although Wang et al [30] report coalescence of grains along what may have been special boundaries. This coalescence would not however lead to absorption of one grain within another to leave only one common WC-Co boundary.

Further studies are clearly required to establish the frequency of the subgrain occurrence as a function of Co volume fraction and grain size and also to investigate the WC-Co boundary orientation relationships where the subgrains have a common WC-Co boundary with the main grain. A more in-depth investigation of the relative sizes and misorientations between subgrains and their enclosing grains would also be worthwhile.

\section{Conclusions}

The combination of an ECCI rotation series and EBSD allows for new insights into the presence of subgrains and dislocations in a WC-Co hardmetal sample over a large, in this case $75 \mu \mathrm{m} \times 75 \mu \mathrm{m}$, field of view. $\mathrm{ECCl}$ exposes dislocation networks within whole grains, $\approx 10-25 \mu \mathrm{m}$ in diameter and can be used to investigate the properties of a large number of grains in a sample. When used in conjunction with EBSD, ECCI is an effective tool for acquiring information on dislocations in undeformed material.

Future work will study and catalogue the dislocations present in [0001] and [11 $\overline{2} 0]$ crystal faces using the techniques described above and investigate the frequency and location of the subgrains observed in many of the grains described in the samples examined here. Information on the subgrain misorientations may also enable some understanding of the mechanism by which they are formed or remain in the sintered structure.

\section{Acknowledgements}

This work was supported by the UK EPSRC project EP/P015719/1,“Quantitative non-destructive nanoscale characterisation of advanced materials".

The data associated with this research are available at [DOI to be inserted] or from the corresponding author.

BMJ acquired the SEM data. BMJ, CTC, KM and AW analyzed the data and wrote the paper together. This work was instigated by NK and KM. BH was consulted for theoretical background. 


\section{References}

[1] V. Randle, Theoretical framework for electron backscatter diffraction, in: A. Schwartz, M. Kumar, B. Adams (Eds.), Electron Backscatter Diffr. Mater. Sci., 2000: pp. 19-30. doi:10.1007/978-14757-3205-4.

[2] R.A. Schwarzer, D.P. Field, B.L. Adams, M. Kumar, A.J. Schwartz, Present State of Electron Backscatter Diffraction and Prospective Developments, in: A.J. Schwartz, M. Kumar, B.L. Adams, D.P. Field (Eds.), Electron Backscatter Diffr. Mater. Sci., Springer US, Boston, MA, 2009: pp. 1-20. doi:10.1007/978-0-387-88136-2_1.

[3] A.J. Wilkinson, P.B. Hirsch, Electron diffraction based techniques in scanning electron microscopy of bulk materials, Micron. 28 (1997) 279-308. doi:10.1016/S0968-4328(97)00032-2.

[4] D.C. Joy, D.E. Newbury, D.L. Davidson, Electron channeling patterns in the scanning electron microscope, J. Appl. Phys. 53 (1982). doi:10.1063/1.331668.

[5] B.A. Simkin, M.A. Crimp, An experimentally convenient configuration for electron channeling contrast imaging, Ultramicroscopy. 77 (1999) 65-75. doi:10.1016/S0304-3991(99)00009-1.

[6] S. Zaefferer, N.N. Elhami, Theory and application of electron channelling contrast imaging under controlled diffraction conditions, Acta Mater. 75 (2014) 20-50. doi:10.1016/j.actamat.2014.04.018.

[7] L.J. Prakash, Application of fine grained tungsten carbide based cemented carbides, Int. J. Refract. Met. Hard Mater. 13 (1995) 257-264. doi:10.1016/0263-4368(95)92672-7.

[8] K.P. Mingard, M.G. Gee, EBSD examination of worn WC/Co hardmetal surfaces, Wear. 263 (2007) 643-652. doi:10.1016/j.wear.2006.11.039.

[9] B. Roebuck, P. Klose, K.P. Mingard, Hardness of hexagonal tungsten carbide crystals as a function of orientation, Acta Mater. 60 (2012) 6131-6143. doi:10.1016/j.actamat.2012.07.056.

[10] B. Roebuck, E. A. Almond, Deformation and Fracture Processes and the Physical Metallurgy of WC-Co Hardmetals, Int. Mater. Rev. 33 (1988) 90-112. doi:10.1179/095066088790324094.

[11] S. Lay, C. H. Allibert, M. Christensen, G. Wahnström, Morphology of WC grains in WC-Co alloys, Mater. Sci. Eng. A. 486 (2008) 253-261. doi:10.1016/j.msea.2007.09.019.

[12] M.A. Yousfi, J. Weidow, A. Nordgren, L.K.L. Falk, H.O. Andrén, Deformation mechanisms in a WCCo based cemented carbide during creep, Int. J. Refract. Met. Hard Mater. 49 (2015) 81-87. doi:10.1016/j.ijrmhm.2014.07.016.

[13] S. Lay, HRTEM investigation of dislocation interactions in WC, Int. J. Refract. Met. Hard Mater. 41 (2013) 416-421. 
[14] U. Farooq, M.U.; Klement, EBSD characterization of carbide - carbide boundaries in WC - Co, J. Microsc. 213 (2004) 306-312.

[15] D.B. Williams, C.B. Carter, Imaging Strain Fields, in: Transm. Electron Microsc. A Textb. Mater. Sci., Springer US, Boston, MA, 2009: pp. 441-461. doi:10.1007/978-0-387-76501-3_26.

[16] G. Naresh-Kumar, B. Hourahine, P.R. Edwards, A.P. Day, A. Winkelmann, A.J. Wilkinson, P.J. Parbrook, G. England, C. Trager-Cowan, Rapid nondestructive analysis of threading dislocations in wurtzite materials using the scanning electron microscope, Phys. Rev. Lett. 108 (2012) 1-5. doi:10.1103/PhysRevLett.108.135503.

[17] G. L'hôte, C. Lafond, P. Steyer, S. Deschanel, T. Douillard, C. Langlois, S. Cazottes, RotationalElectron Channeling Contrast Imaging analysis of dislocation structure in fatigued copper single crystal, Scr. Mater. 162 (2019) 103-107. doi:10.1016/j.scriptamat.2018.10.050.

[18] R. Hielscher, H. Schaeben, A novel pole figure inversion method: specification of the MTEX algorithm, J. Appl. Crystallogr. 41 (2008) 1024-1037. doi:10.1107/S0021889808030112.

[19] F. Bachmann, R. Hielscher, H. Schaeben, Texture Analysis with MTEX - Free and Open Source Software Toolbox, Solid State Phenom. 160 (2010) 63-68. doi:10.4028/www.scientific.net/ssp.160.63.

[20] G. Nolze, R. Hielscher, Orientations - perfectly colored, J. Appl. Crystallogr. 49 (2016) 1786-1802. doi:10.1107/S1600576716012942.

[21] G. Nolze, R. Hielscher, A. Winkelmann, Electron backscatter diffraction beyond the mainstream, Cryst. Res. Technol. 52 (2017) 1-24. doi:10.1002/crat.201600252.

[22] S.I. Wright, M.M. Nowell, D.P. Field, A Review of Strain Analysis Using Electron Backscatter Diffraction, Microsc. Microanal. 17 (2011) 316-329. doi:10.1017/s1431927611000055.

[23] J. Edington, Monograph 3, Interpretation of Transmission Electron Micrographs, in: Pract. Electron Microsc. Mater. Sci., Eindhoven: Philips Technical Library, 1976.

[24] E. Pascal, B. Hourahine, G. Naresh-Kumar, K. Mingard, C. Trager-Cowan, Dislocation contrast in electron channelling contrast images as projections of strain-like components, Mater. Today Proc. 5 (2018) 14652-14661. doi:10.1016/j.matpr.2018.03.057.

[25] B.H. Kong, Q. Sun, J. Han, I.H. Lee, H.K. Cho, Classification of stacking faults and dislocations observed in nonpolar a-plane GaN epilayers using transmission electron microscopy, Appl. Surf. Sci. 258 (2012) 2522-2528. doi:10.1016/j.apsusc.2011.10.086.

[26] S. Hagege, J. Vicens, G. Nouet, P. Delavignette, Analysis of structure defects in tungsten carbide, Phys. Status Solidi. 61 (1980) 675-687. doi:10.1002/pssa.2210610242. 
[27] M.K. Hibbs, R. Sinclair, Room-temperature deformation mechanisms and the defect structure of tungsten carbide, Acta Metall. 29 (1981) 1645-1654. doi:https://doi.org/10.1016/00016160(81)90047-X.

[28] A. Delanoë, S. Lay, J.-M. Missiaen, Microstructure Features of Large Grains in WC-Co Alloys, Mater. Sci. Forum - MATER SCI FORUM. 534-536 (2007) 1213-1216. doi:10.4028/www.scientific.net/MSF.534-536.1213.

[29] M. Pellan, S. Lay, J.M. Missiaen, S. Norgren, J. Angseryd, E. Coronel, T. Persson, Effect of Binder Composition on WC Grain Growth in Cemented Carbides, J. Am. Ceram. Soc. 98 (2015) 35963601. doi:10.1111/jace.13748.

[30] X. Wang, Z.Z. Fang, H.Y. Sohn, Grain growth during the early stage of sintering of nanosized WCCo powder, Int. J. Refract. Met. Hard Mater. 26 (2008) 232-241. doi:10.1016/j.ijrmhm.2007.04.006.

[31] Y. Zhong, H. Zhu, L.L. Shaw, R. Ramprasad, The equilibrium morphology of WC particles - A combined ab initio and experimental study, Acta Mater. 59 (2011) 3748-3757. doi:10.1016/j.actamat.2011.03.018.

[32] S. Kim, S.H. Han, J.K. Park, H.E. Kim, Variation of WC grain shape with carbon content in the WCCo alloys during liquid-phase sintering, Scr. Mater. 48 (2003) 635-639. doi:10.1016/S13596462(02)00464-5.

[33] V. Bounhoure, S. Lay, F. Charlot, A. Antoni-Zdziobek, E. Pauty, J.M. Missiaen, Effect of C content on the microstructure evolution during early solid state sintering of WC-Co alloys, Int. J. Refract. Met. Hard Mater. 44 (2014) 27-34. doi:10.1016/j.jjrmhm.2013.12.012. 